\title{
SMA Actuated Finger Exoskeleton Device for Rehabilitation of Acute Paresis Patient
}

\author{
Abdul Hakim Ab. Rahim ${ }^{1, a}$, Andreas Lachmann ${ }^{2, b}$, Cheng Yee Low ${ }^{1, c}$ \\ and Adam Tan Mohd Amin ${ }^{1, d}$ \\ ${ }^{1}$ Faculty of Mechanical Engineering, Universiti Teknologi MARA, 40450 Shah Alam, Malaysia \\ ${ }^{2}$ Fachhochschule Brandenburg University of Applied Sciences, Magdeburger Str.50, 14776 \\ Brandenburg an der Havel, Germany \\ ahakimrahim@gmail.com, 'lachmana@fh-brandenburg.de, 'chengyee.low@salam.uitm.edu.my, \\ adam.tan86@gmail.com
}

Keywords: Finger rehabilitation, exoskeleton, Shape Memory Alloys, acute stroke

Abstract. Functional recovery of upper limb after stroke is crucial to restore the ability to perform activities of daily living (ADL). This paper presents a robotic rehabilitation approach based on repetitive exercise aimed to help stroke survivors relearn the skills of finger flexion and extension at the comfort of their home. The finger rehabilitation device deploys Shape Memory Alloy (SMA) wires as an actuation approach to deliver three degrees of freedom per finger module. The advantages and challenges of using SMA wires rather than conventional actuators are discussed. A prototype of the finger rehabilitation device was built using PLA material and experiments have been conducted for the purposes of feasibility study. Tests conducted on the wires suggest that it has to have sufficient weight and also has to be stretching at high temperature rather than room temperature in order to have an optimum range of recovery.

\section{Introduction}

Robotic approach in human limbs rehabilitation is not a new subject discussed among researchers and therapist. The interest on this topic ranged from the best design suitable for human morphology to the best design that can provide beneficial movements and improvements for the patient. The SMA has been introduced in the robotic arena since 1980's and until today. The development of the SMA to be used as the perfect actuator is still in an ongoing process[1]. SMA is a metallic material consists of several substances that have the ability to change to its predetermined shape or size when exposed to a thermal condition. This smart material have many kind of polycrystalline combinations, but the Nickel-Titanium (NiTi)is considered to have the most beneficial properties to be used as an actuator [2] among other actuators such as electric motor, electromagnet, pneumatic cylinders or hydraulic.

The SMA 'memorizes' its shape because of the changes of its crystalline structure from austenite, highly symmetrical to martensite which is in less symmetrical order and vice versa. In initial low temperature (stable martensitic phase), the SMA's shape can be deformed plastically and be heated to a high temperature for it to return to its original shape (stable austenite phase)[1]. The transformation temperature of an SMA wire starts at $52.54{ }^{\circ} \mathrm{C}$ and ends at $60.90{ }^{\circ} \mathrm{C}$ for austenite while for martensite starts at $44.78{ }^{\circ} \mathrm{C}$ and ends at $32.84{ }^{\circ} \mathrm{C}$ although it is actually depends on the weight condition [3].

Because of these feature, SMA wires have many advantages to be used as an actuator. It has high force to weight ratio with a significant reduction in sizes and complexity compared to other bulky actuator besides silent and smooth operations among many others [1]. It can react to environmental stimuli which can be its advantage or challenge [3,4] depending on the objective of the design. Other challenges includes its small usable strain which is about $4 \%$ based on its Recommended Recovery Ratio (RRR) [5], low accuracy and low energy efficiency. These challenges however did not stop many researchers to use the SMA wires in their design. The effort of using SMA wire in robotic application especially in artificial hands for manipulating objects were numerous and starts 
as early as 1980s [6] until today[3,6,7]. This paper presents the project Cable Actuated Finger Exoskeleton (CAFEx) which uses the SMA wire in an exoskeleton for finger as a rehabilitation device for acute stroke paralyzed survivor. Although the working concepts of these devices are about the same, the procedures that the device must deliver are different. While artificial hands need to take or grab object with weights, CAFEx works as a supporting device for patient to move their finger to certain positions. CAFEx must follow the exercise routine suggested by rehabilitation therapist to ensure improvements for patient's finger health. The objective is to provide an extension-flexion movement for the finger of an acute paralyzed patient. A paper on the mechanical design of CAFEx was presented in 2013 [9] but on this paper only the actuation system was originally designed for it while the testing on the other hand was done using the design of Handexos from other researcher.

\section{Mechanical Structure / Material Modification}

HANDEXOS[10] are being used as the fundamental study in order to further understand the problem because the design is robust and tested on a normal person's hand. However some modifications are proposed in this project specifically to suit the device with the current objective. This project is focused only on the fingers. This is due to the fact that the thumb has much more complicated range of motion compared to the rest. A prototype of HANDEXOS was fabricated using 3D printer The Replicator using PLA material (Fig.1). PLA material is also suggested to be used as the final product material because it is easy to be fabricated, cheap and light. However the area that interacts with the tendon cable seems to be wearing fast caused by the friction. These components need to be made by a sturdier material such as metallic material or strong plastic with perfect finishing.

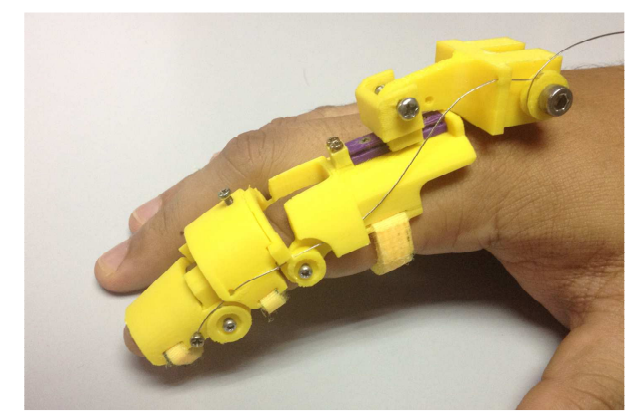

Figure 1: A rebuild HANDEXOS prototype using PLA with tendon wire and Velcro strap

A layer of polyurethane gel was used as the inner surface of the module to solve the fixation issue that was raised when the previous paper was presented. This gel is quite tacky to hold the finger fixed in positions while backed up with Velcro straps when the device operates.

The tendon wire (steel wire) was positioned trough the finger module pulleys, and connected to the SMA wire at a hand base as the actuation system. All finger modules were placed on the hand base module while the actuation systems were placed on an arm base. CAFEx was designed to be used on a table on top of an arm holder (Fig. 2(a)) designed to give a support base for the exercise process. The patient needs to put the thumb under the horizontal cylinder to be in a natural position while the rest of the fingers are being exercised.

\section{Actuation Analysis}

Referring to the results of HANDEXOS [9] the maximum tendon cable force is $150 \mathrm{~N}$. To reach this force of movement FLEXINOL ${ }^{\circledR}$ Actuator Wire with a diameter of $0.88 \mathrm{~mm}$ is needed. However, for the amount of force needed to move acute paralyzed finger of a patient is much less than that. Assuming the finger has minimal to none spacticity, the only contributing weights are the weights of the finger and modules which is estimated to be less than $8 \mathrm{~N}$. An SMA wire with diameter of 
$0.25 \mathrm{~mm}$ is used in this project based on the maximum force it can provide at $8.91 \mathrm{~N}$. The project can proceed further with bigger wire diameter for higher load.
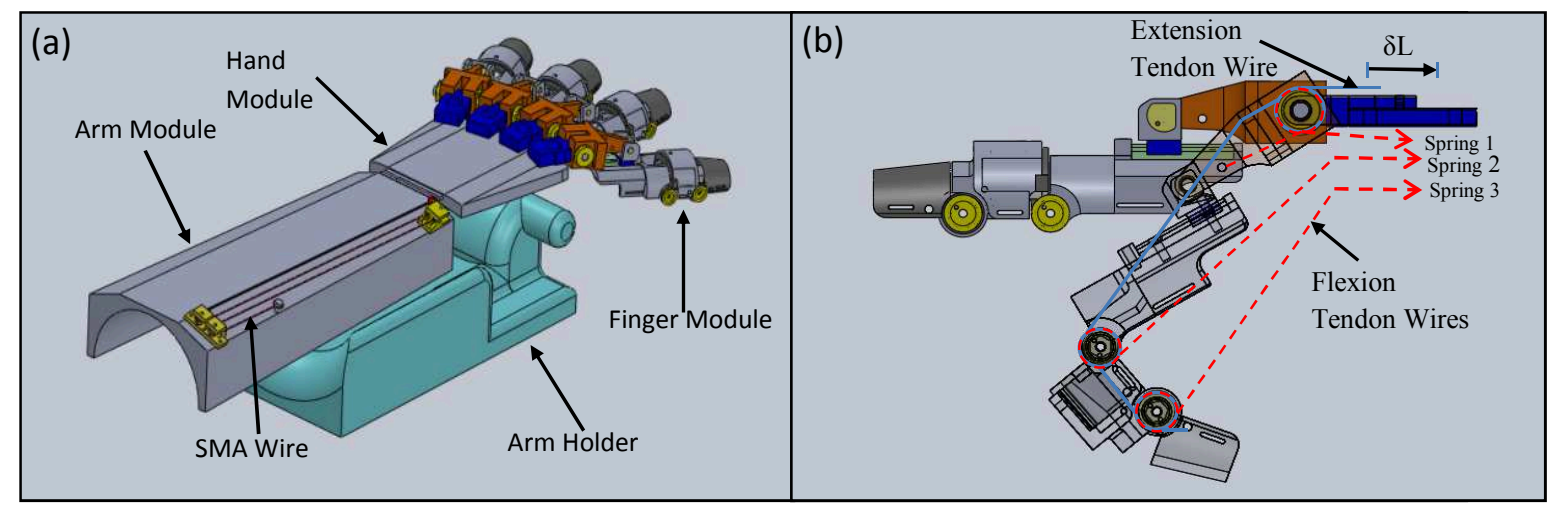

Figure 2 :(a) Full view of a left-handed CAFEx Modules with SMA wire in "M-Shape" arrangement; (b) CAFEx finger module in full extension and flexion mode

The exercise's objective is to bring one's finger from a flexion position to an extension position. Extension movement will be provided by the SMA wire whereas the flexion movement was set as the default position and force from preloaded springs will bring the finger back to flexion from extension position. After a calculation for one specific finger for initial study, the tendon wire needs to be retracted by an amount of $28 \mathrm{~mm}(\delta \mathrm{L})$ to deliver a full extension (Fig. 2(b)). This means an actuation system that can provide a pull or a linear movement of that amount is needed. For an SMA wire that can only offer a maximum movement of $4 \%$ of its length means $700 \mathrm{~mm}$ of SMA wire need to be planted on the arm module. SMA wire can be organized on the arm module with help of some small bearings. The SMA wire was planted in an "M-shape" (Fig. 2(a)) arrangement resulting only less than $1 / 3$ area of its original length required.

Due to the displacement of the SMA wire the electrical resistance changed so much that it is possible to use this effect as a sensor value for electronically controlled systems. By changing the electrical resistance all intermediate positions could be adjusts with high control precision. A displacement sensor is not necessary. The SMA wire was controlled by a motor driver using a pulse width modulation signal and a microcontroller, tested with dead weight hanging freely at the end with temperature of the room and the wire was recorded. The driver directs current from power source to the SMA wire from one channel and to the fans from the other channel.

Three experiments were conducted to see the relation between SMA Wire contraction range vs weights i.e. the relation when the temperature of the wire is cool (room temperature); the relation when the wire temperature is hot (transformation temperature); and lastly the relation when the wire is hot but cooled down rapidly with the aid of air flowing (fan). The tests were done by providing weights to pull the SMA wire in hot/cold situation so that it will change from austenitic to martensitic phase and have the potential displacement (contraction range). Experimental setup is shown in Fig. 3.

This device intended to be used by any patient. This means that different weights of fingers will be put on the same setting of actuator. Even though this is acceptable if the weight did not exceed the maximum capacity, it will affect the performance of the actuator. To properly understand this situation, first experiment was conducted to see how weight influences the optimum contraction range. The result in Figure 4(a) shows that higher weight produces higher potential displacement. This also means if there were not enough weight on the device, the SMA wire would not have an optimum range. This situation is also related to time-joint spasticity issues as patient's finger will become harder to move by time which can be interpreted as a weight factor for the actuator. 


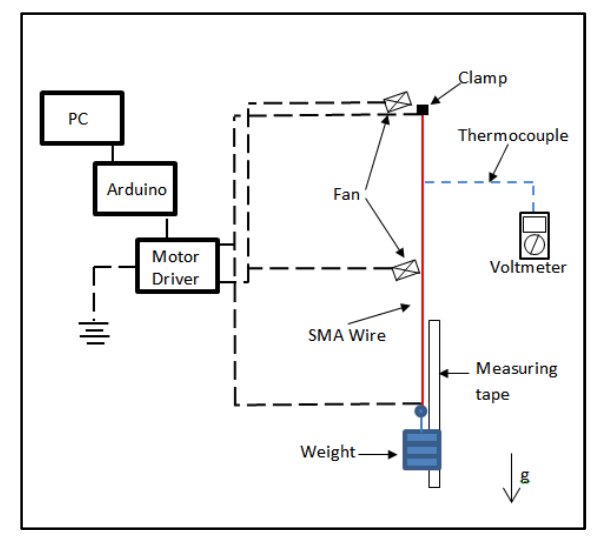

Figure 3: Experimental setup of SMA wire

With this property of the SMA wire means in order to have an optimum range of extension and flexion of patient's finger, the device have to always be pre-set to offer enough weight before operation. Assuming patient's finger has the weight of less than $100 \mathrm{~g} /$ finger, it can be concluded that all the balance weight has to be supplied by the force from the flexion springs. The device must have the option to set the value of force supplied by the spring before working.

The second condition needed to be fulfill by the device is that a set of exercise for one's finger will have to be conducted in a timely manner, meaning that the device may need to extends the finger and holds it at that position for some time before releasing it. However, using currentsupplied heating for the SMA wire will affect the energy efficiency especially when battery is intended to be used as a portable power source solution. One of the proposed solutions is to provide a mechanical stopper or locking mechanism [8] to hold the finger on extended mode before releases it. This means the current supply for the actuator will be cut off while holding at certain position. The second experiment shows that the SMA wire will not have an optimum contraction range if it pulled at cool (room) temperature. Fig 4(b) shows the difference between hot operated and cool operated wire displacement range.
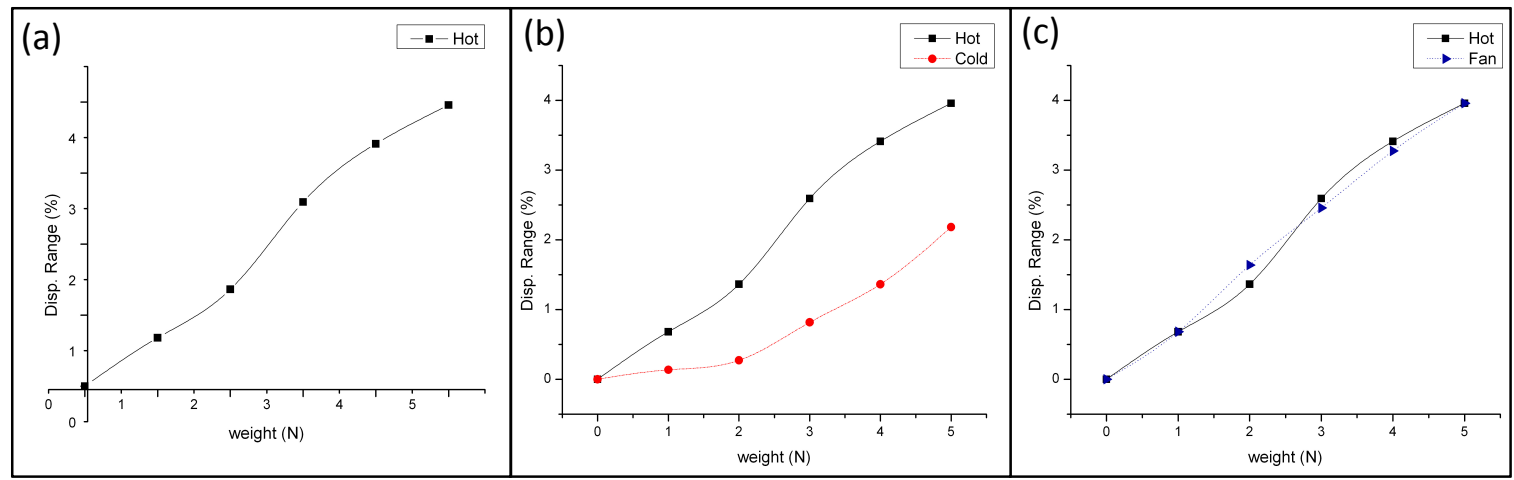

Figure 4: Graphs showing the result of all 3 experiments displacement range- weight relation. (a) Controlled hot wire temperature. (b) Comparison between hot and cold wire initial temperature. (c) Comparison between with and without fan.

There were a large different of contraction range between cool and hot pulled SMA wire. Operating the wire in cool temperature 'reserves' the wire from providing the optimal displacement range for the device compared to hot temperature. Another experiment was conducted with the weight pulled SMA wire in hot condition but cooled rapidly to room temperature using the same apparatuses with addition of fans to provide air ventilation on the wire. It turns out that the actuator behaves on the same way as the hot operated actuator (Fig. 4(c)) with less time to reach its maximum displacement range was observed. 


\section{Conclusion}

The actuator for CAFEx is discussed in this paper. The actuation system and mechanical parts works well to provide flexion and extension for the exoskeleton prototype. Based on the experiments done, it can be concluded that the spring setup at the flexion unit should be set to provide enough force for the SMA wire to produce its contraction range at the recommended value which is $4 \%$. There is also significant efficiency reduction of contraction range of the SMA wire if it is operated in cool temperature. There are several other experiment and tests to be conducted and will be reported in the future.

\section{Acknowledgement}

The authors thank Universiti Teknologi MARA, the Ministry of Science, Technology and Innovation Malaysia [100-RMI/SF 16/6/2 (3/2014)] and the Ministry of Education Malaysia [600RMI/ERGS 5/3 (15/2013) and 600-RMI/FRGS 5/3 (77/2014)] for funding the research work.

\section{Reference}

[1] J. Mohd Jani, M. Leary, A. Subic, and M. A. Gibson, "A review of shape memory alloy research, applications and opportunities," Mater. Des., vol. 56, pp. 1078-1113, Apr. 2014.

[2] W. Huang, "On the selection of shape memory alloys for actuators," no. June 2000, pp. 1119, 2002.

[3] T. W. Choon, A. S. Salleh, S. Jamian, and M. I. Ghazali, "Phase Transformation Temperatures for Shape Memory Alloy Wire,” pp. 304-307, 2007.

[4] C. Yee, M. A. A. Kasim, T. Koch, R. Dumitrescu, H. Yussof, R. Jaafar, A. Jaffar, A. Aqilah, and K. Mun, "Hybrid-Actuated Finger Prosthesis with Tactile Sensing," Int. J. Adv. Robot. Syst., p. 1, 2013.

[5] M. Vasina, F. Solc, and K. Hoder, "Shape memory alloys - unconventional actuators," in IEEE International Conference on Industrial Technology, 2003, 2003, vol. 1, pp. 190-193.

[6] M. Bergamasco, F. Salsedo, and P. Dario, "Shape memory alloy micromotors for direct-drive actuation of dexterous artificial hands," Sensors and Actuators, vol. 17, no. 1, pp. 115-119, 1989.

[7] V. Farias, L. Solis, L. Melendez, C. Garcia, and R. Velazquez, "A four-fingered robot hand with shape memory alloys," in AFRICON 2009, 2009, pp. 1-6.

[8] K. Andrianesis and A. Tzes, "Design of an anthropomorphic prosthetic hand driven by Shape Memory Alloy actuators," in 2008 2nd IEEE RAS \& EMBS International Conference on Biomedical Robotics and Biomechatronics, 2008, pp. 517-522.

[9] A. H. Ab Rahim, M. N. A. Bin Ab Patar, A. T. M. Amin, and J. Mahmud, "The Development of Finger Rehabilitation Device for Stroke Patients," Appl. Mech. Mater., vol. 393, pp. 604 610, Sep. 2013.

[10] A. Chiri, N. Vitiello, F. Giovacchini, S. Roccella, F. Vecchi, and M. C. Carrozza, "Mechatronic Design and Characterization of the Index Finger Module of a Hand Exoskeleton for Post-Stroke Rehabilitation," IEEE/ASME Trans. Mechatronics, vol. 17, no. 5, pp. 884-894, Oct. 2012. 